\title{
Investigation on Fabrication of SS316 Gear by Wire-EDM
}

\author{
Kapil Gupta ${ }^{1, *}$ \\ ${ }^{1}$ Mechanical and Industrial Engineering Technology, University of Johannesburg, 2028 Johannesburg, \\ South Africa
}

\begin{abstract}
Manufacturing of miniature gears with good accuracy and quality, and at low cost is a challenging task for traditional manufacturing processes. Wire-cut electric discharge machining (WEDM) has the capacity to machine any conductive material to fabricate typical shaped products like gears with high level of accuracy and quality. In present research, stainless steel (SS) alloy 316 is used to fabricate miniature gears by WEDM. The experimental array is designed according to Response Surface Methodology (RSM) based central composite face centered (CCFC). Four input parameters namely servo feed (SF), duty factor (DF), servo voltage (SV) and wire feed (WF) with three levels each are used for experimental array to investigate the process performance in terms of cutting rate (CR), and product i.e. gear quality in terms of mean roughness depth $(\mathrm{Rz})$ and dimensional deviation (DD). After WEDM parameter optimization, confirmation experiments are performed at the parametric setting by considering equal importance to all response variables. A very close agreement between the experimental and predicted values have been observed.
\end{abstract}

\section{Introduction}

Gear is a machine component having teeth cut on its periphery. It is a key element in motion and/or power transmission devices irrespective of their sizes. Small size gears, generally known as miniature gears are extensively used in appliances, scientific instruments, equipment, and speed reducers $[1,2]$. Plastics, brass, stainless steel, and bronze are most common materials for small gear manufacturing. Stainless steel (SS) 316 small gears are mainly used for transmission of torque in miniature devices. The quality of gear tooth surfaces, and dimensional and geometrical accuracy are important from the point of view of their performance during their application where they are employed. Conventional manufacturing of these gears is done by stamping, hobbing, milling, powder metallurgy, and forging $[2,3]$. But, several challenges are encountered during conventional manufacturing of gears due to the inherent limitations of the processes and gear material properties. Surface quality, dimensional and geometrical accuracy, as obtained by these processes are of low grade, and gears need to be exposed to post-finishing or treatment operations [4]. Which mainly escalates the overall manufacturing cost and time. An alternate is to try fabricating

\footnotetext{
*Corresponding author: kgupta@uj.ac.za
} 
them by advanced machining processes like electric discharge machining, laser beam machining, and abrasive water jet machining techniques.

There has been few previous research work on manufacturing of small size gears by these processes [4-8]. From the point of view of process productivity, laser machining has been identified as the most superior processes. Whereas from sustainability point of view, abrasive water jet machining process has been identified as the best process. After reviewing the literature, a dearth of research is observed on the development of miniature gear of SS316 alloy by WEDM. The work reported in this paper, attempts to fulfil this gap. This paper discusses the wire-EDM based fabrication of SS316 miniature gear and presents the details of results.

\section{Experimental Methodology}

A rectangular plate $(150 \mathrm{~mm} \times 155 \mathrm{~mm} \times 5 \mathrm{~mm})$ of SS316 alloy is used for the manufacturing of miniature gears. SS316 exhibit a good corrosion resistance with high strength. The outside diameter of gear is kept at $9 \mathrm{~mm}$ with 10 teeth. Wire-electrical discharge machine of Electronica (Pune, India) make Ecocut (Elplus 15) is used for the preparation of miniature gears. Zinc coated brass wire $(250 \mu \mathrm{m}$ diameter) is used as an electrode in WEDM. The deflections in the wire are kept at its minimum by selecting an appropriate value of wiretension. Deionized water is utilized as a dielectric to remove the micro-chips of machine material from the spark-gap. The activities occurred in current research in form of flow diagram is depicted in Fig. 1. In the present research, four process parameters are varied according to the experimental array. The range of working parameters are identified after some preliminary experiments. The detail of working parameters and constant parameters are provided in Table 1.

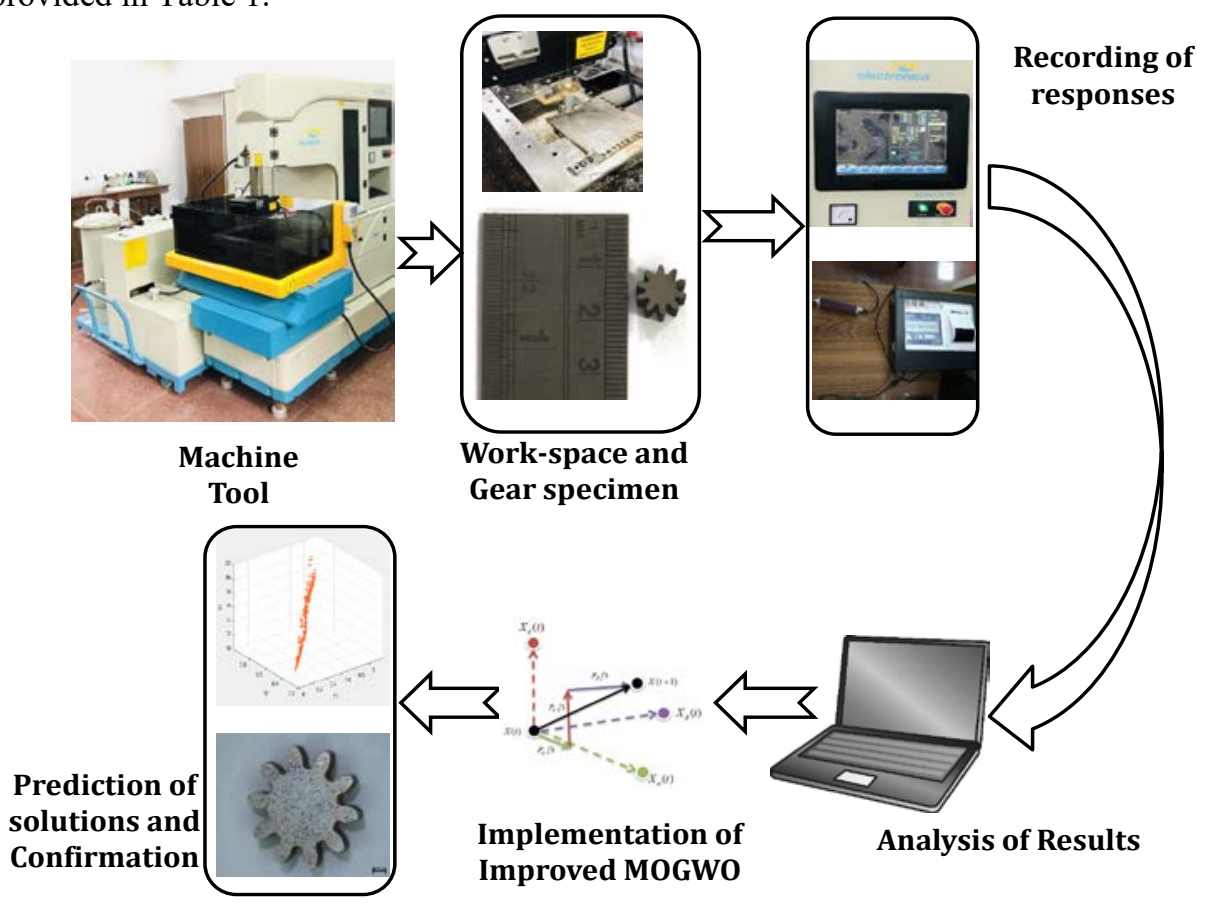

Fig. 1. Overview of process flow in WEDM based fabrication of gears in the present work. 
Table 1. WEDM process parameter details

\begin{tabular}{|c|c|c|c|c|}
\hline \multicolumn{5}{|c|}{ Variable process parameters } \\
\hline Process Parameter & Notation & Unit & Lower Limit & Higher limit \\
\hline Servo Feed & SF & $\mathrm{mm} / \mathrm{min}$ & 1000 & 1900 \\
\hline Duty Factor & $\mathrm{DF}$ & - & 0.6 & 0.75 \\
\hline Servo Voltage & $\mathrm{SV}$ & $\mathrm{V}$ & 30 & 50 \\
\hline Wire Feed & $\mathrm{WF}$ & $\mathrm{m} / \mathrm{min}$ & 4 & 12 \\
\hline \multicolumn{2}{|c|}{ Fixed process parameters } \\
\hline Workpiece & \multicolumn{3}{|c|}{$\mathrm{SS} 316$ alloy } \\
\hline Dielectric temperature & $20-24^{\circ} \mathrm{C}$ \\
\hline Dielectric & Deionized water \\
\hline Working temperature & $25^{\circ} \mathrm{C}$ \\
\hline
\end{tabular}

The design of experiments in current research is based on face centered central composite technique of response surface methodology [9]. In this, four control factors having three levels (low, medium and high) each are varied to investigate their effects on response characteristics. A total of 30 experiments were conducted as per the suggested settings and each experiment was repeated twice (i.e. two replications for every experiment) to minimize the experimental error. Cutting rate $(\mathrm{CR})$, mean roughness depth $(\mathrm{Rz})$ and dimensional deviation (DD) are the outputs or response characteristics investigated in the present research. $\mathrm{CR}$ was directly investigated from the machine tool monitor. The measurement of $\mathrm{Rz}$ is made with the help of Mitutoyo make Surftest (SJ-410P, least count- $0.001 \mu \mathrm{m}$ ). For the measurement of dimensional deviation, the outside diameter is considered. As the research is conducted for $9 \mathrm{~mm}$ diameter gears. Therefore, any deviation from this value will cause the dimensional deviation. To investigate the dimensional deviation, digital micrometer (Mitutoyo make, least count- $0.01 \mu \mathrm{m}$ ) is used. The optical micrographs of the miniature gears were captured by Carl-Zeiss make optical microscope.

\section{Results and Discussion}

Table 2 demonstrates the experimental array of the input parameters along with the corresponding values of response characteristics. The statistical summary of the pooled ANOVAs for CR, Rz and DD is given in Table 3. It is evident from the first part of the analysis that terms like SF, DF, SV, WF, interaction terms of DF and SV, quadratic terms of $\mathrm{SF}$ and DF have the influential effect on the analysis of CR. These terms played influential role for the investigation of CR due to P-value less than 0.05 . The non-significant value of lack of fit and significant value of model suggests good ANOVA. High F-value corresponds to the larger contribution of input parameter for the analysis of CR. During the analysis of $\mathrm{Rz}$, it is clear that there is no interaction term which is significant, however the quadratic terms of SF and DF plays pivotal contribution. The terms SF, DF and WF also play crucial role for the investigation of $\mathrm{Rz}$ due to P-value less than 0.05 . All the process parameters, interaction term of SF and DF, quadratic term of SF and WF plays significant role for the analysis of DD (as pe the ANOVA of DD). A sign of good ANOVA is observed as lack of fit is not significant and model is significant. The values of MS in all cases can be evaluated by dividing the SS of parameter to corresponding degree of freedom (dof).

Fig. 2 shows the variation of response variable (CR, Rz and DD) with the input process parameters (SF, DF, SV and WF). Fig. 2a shows that the maximum $\mathrm{CR}$ is obtained at maximum value of SF $(1900 \mathrm{~mm} / \mathrm{min})$. It is observed from Fig. $2 b$ that the minimum value of $\mathrm{Rz}$ and $\mathrm{DD}$ is found at SF $(1450 \mathrm{~mm} / \mathrm{min})$. With the increase in DF value the CR and $\mathrm{Rz}$ increases, however the DD decreases. DF is the ratio of pulse duration to the total cycle time value (total cycle time $=$ pulse on time + pulse off time). All the responses are measured at 
three values of DF $(0.6,0.67$ and 0.75$)$. It is observed that smaller value of DF shows a greater stability in WEDM operation and therefore smaller valuer of $\mathrm{CR}, \mathrm{Rz}$ and $\mathrm{DD}$ is obtained. This happens due to small discharge frequency at smaller value of DF. Also, low level of contamination in the spark gap is observed at low DF. With increase in the DF (up to 0.75), delay in the ignition time decreases which sets up a bridge in the spark gap and intensify the electric field. The reason for this is the adherence of debris particles in the machining gap.

Table 2. Experimental array and corresponding responses.

\begin{tabular}{|c|c|c|c|c|c|c|c|c|}
\hline Std & Run & SF & DF & SV (V) & $\begin{array}{c}\text { WF } \\
(\mathbf{m} / \mathbf{m i n})\end{array}$ & $\begin{array}{c}\text { CR } \\
(\mathrm{mm} / \mathrm{min})\end{array}$ & $\operatorname{Rz}(\mu \mathrm{m})$ & DD (\%) \\
\hline 2 & 1 & 1900 & 0.6 & 30 & 4 & 0.3 & 15.795 & 0.443 \\
\hline 13 & 2 & 1000 & 0.6 & 50 & 12 & 0.39 & 15.391 & 0.497 \\
\hline 18 & 3 & 1900 & 0.68 & 40 & 8 & 0.79 & 19.497 & 0.717 \\
\hline 20 & 4 & 1450 & 0.75 & 40 & 8 & 0.85 & 14.943 & 0.778 \\
\hline 5 & 5 & 1000 & 0.6 & 50 & 4 & 0.21 & 13.862 & 0.346 \\
\hline 23 & 6 & 1450 & 0.68 & 40 & 4 & 0.4 & 15.445 & 0.517 \\
\hline 22 & 7 & 1450 & 0.68 & 50 & 8 & 0.43 & 15.996 & 0.59 \\
\hline 11 & 8 & 1000 & 0.75 & 30 & 12 & 0.89 & 20.007 & 0.803 \\
\hline 26 & 9 & 1450 & 0.68 & 40 & 8 & 0.45 & 16.293 & 0.576 \\
\hline 15 & 10 & 1000 & 0.75 & 50 & 12 & 0.8 & 19.332 & 0.73 \\
\hline 30 & 11 & 1450 & 0.68 & 40 & 8 & 0.47 & 16.613 & 0.581 \\
\hline 16 & 12 & 1900 & 0.75 & 50 & 12 & 0.85 & 19.979 & 0.79 \\
\hline 1 & 13 & 1000 & 0.6 & 30 & 4 & 0.26 & 14.991 & 0.374 \\
\hline 9 & 14 & 1000 & 0.6 & 30 & 12 & 0.37 & 15.193 & 0.477 \\
\hline 6 & 15 & 1900 & 0.6 & 50 & 4 & 0.27 & 15.112 & 0.383 \\
\hline 25 & 16 & 1450 & 0.68 & 40 & 8 & 0.46 & 17.178 & 0.614 \\
\hline 8 & 17 & 1900 & 0.75 & 50 & 4 & 0.77 & 19.119 & 0.703 \\
\hline 12 & 18 & 1900 & 0.75 & 30 & 12 & 0.95 & 20.919 & 0.997 \\
\hline 14 & 19 & 1900 & 0.6 & 50 & 12 & 0.44 & 16.069 & 0.558 \\
\hline 17 & 20 & 1000 & 0.68 & 40 & 8 & 0.73 & 18.673 & 0.683 \\
\hline 4 & 21 & 1900 & 0.75 & 30 & 4 & 0.91 & 18.876 & 0.823 \\
\hline 7 & 22 & 1000 & 0.75 & 50 & 4 & 0.71 & 18.492 & 0.623 \\
\hline 21 & 23 & 1450 & 0.68 & 30 & 8 & 0.58 & 16.264 & 0.617 \\
\hline 28 & 24 & 1450 & 0.68 & 40 & 8 & 0.44 & 16.463 & 0.573 \\
\hline 24 & 25 & 1450 & 0.68 & 40 & 12 & 0.49 & 16.942 & 0.597 \\
\hline 10 & 26 & 1900 & 0.6 & 30 & 12 & 0.41 & 15.832 & 0.55 \\
\hline 29 & 27 & 1450 & 0.68 & 40 & 8 & 0.4 & 15.354 & 0.549 \\
\hline 19 & 28 & 1450 & 0.6 & 40 & 8 & 0.23 & 12.316 & 0.362 \\
\hline 27 & 29 & 1450 & 0.68 & 40 & 8 & 0.48 & 16.711 & 0.584 \\
\hline 3 & 30 & 1000 & 0.75 & 30 & 4 & 0.87 & 18.613 & 0.782 \\
\hline
\end{tabular}


Table 3. Pooled ANOVA for CR, Rz, and DD

\begin{tabular}{|c|c|c|c|c|c|c|}
\hline \multicolumn{7}{|c|}{ Pooled ANOVA for CR } \\
\hline Source & SS & dof & MS & F-Value & Prob $>$ F & \\
\hline Model & 1.5 & 7 & 0.21 & 87.3 & $<0.0001$ & significant \\
\hline A-SF & 0.012 & 1 & 0.012 & 4.8 & 0.0394 & \\
\hline B-DF & 1.24 & 1 & 1.24 & 504.93 & $<0.0001$ & \\
\hline C-SV & 0.025 & 1 & 0.025 & 10.17 & 0.0042 & \\
\hline D-WF & 0.044 & 1 & 0.044 & 17.95 & 0.0003 & \\
\hline BC & 0.013 & 1 & 0.013 & 5.4 & 0.0298 & \\
\hline $\mathbf{A}^{\wedge} 2$ & 0.15 & 1 & 0.15 & 61.68 & $<0.0001$ & \\
\hline $\mathrm{D}^{\wedge} 2$ & 0.038 & 1 & 0.038 & 15.64 & 0.0007 & \\
\hline Residual & 0.054 & 22 & $2.45 \mathrm{E}-03$ & & & \\
\hline Lack of Fit & 0.05 & 17 & $2.94 \mathrm{E}-03$ & 3.67 & 0.078 & $\begin{array}{c}\text { not } \\
\text { significant }\end{array}$ \\
\hline Pure Error & $4.00 \mathrm{E}-03$ & 5 & $8.00 \mathrm{E}-04$ & & & \\
\hline Cor Total & 1.55 & 29 & & & & \\
\hline \multicolumn{7}{|c|}{ Pooled ANOVA for $R z$} \\
\hline Source & SS & dof & MS & F-Value & Prob $>$ F & \\
\hline Model & 114.85 & 5 & 22.97 & 89.46 & $<0.0001$ & significant \\
\hline A-SF & 2.45 & 1 & 2.45 & 9.55 & 0.005 & \\
\hline B-DF & 70.88 & 1 & 70.88 & 276.07 & $<0.0001$ & \\
\hline D-WF & 4.87 & 1 & 4.87 & 18.95 & 0.0002 & \\
\hline $\mathbf{A}^{\wedge} \mathbf{2}$ & 36.49 & 1 & 36.49 & 142.12 & $<0.0001$ & \\
\hline $\mathrm{B}^{\wedge} 2$ & 16.68 & 1 & 16.68 & 64.98 & $<0.0001$ & \\
\hline Residual & 6.16 & 24 & 0.26 & & & \\
\hline Lack of Fit & 4.31 & 19 & 0.23 & 0.61 & 0.7997 & $\begin{array}{c}\text { not } \\
\text { significant }\end{array}$ \\
\hline Pure Error & 1.85 & 5 & 0.37 & & & \\
\hline Cor Total & 121.01 & 29 & & & & \\
\hline \multicolumn{7}{|c|}{ Pooled ANOVA for DD } \\
\hline Source & SS & dof & MS & F-Value & Prob $>$ F & \\
\hline Model & 0.66 & 7 & 0.094 & 86.76 & $<0.0001$ & significant \\
\hline A-SF & 0.023 & 1 & 0.023 & 21.58 & 0.0001 & \\
\hline B-DF & 0.51 & 1 & 0.51 & 473.1 & $<0.0001$ & \\
\hline C-SV & 0.023 & 1 & 0.023 & 21.38 & 0.0001 & \\
\hline D-WF & 0.056 & 1 & 0.056 & 51.74 & $<0.0001$ & \\
\hline BC & 0.016 & 1 & 0.016 & 14.35 & 0.001 & \\
\hline$A^{\wedge} 2$ & 0.027 & 1 & 0.027 & 24.65 & $<0.0001$ & \\
\hline$D^{\wedge} 2$ & 0.01 & 1 & 0.01 & 9.57 & 0.0053 & \\
\hline Residual & 0.024 & 22 & $1.09 \mathrm{E}-03$ & & & \\
\hline Lack of Fit & 0.022 & 17 & $1.27 \mathrm{E}-03$ & 2.9 & 0.1216 & $\begin{array}{c}\text { not } \\
\text { significant }\end{array}$ \\
\hline Pure Error & $2.20 \mathrm{E}-03$ & 5 & $4.40 \mathrm{E}-04$ & & & \\
\hline Cor Total & 0.68 & 29 & & & & \\
\hline
\end{tabular}




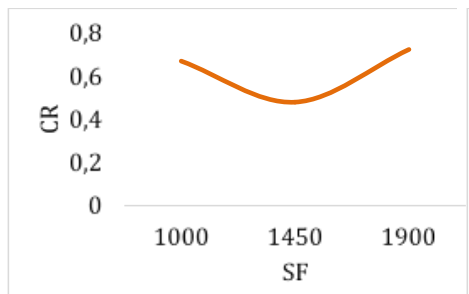

(a)

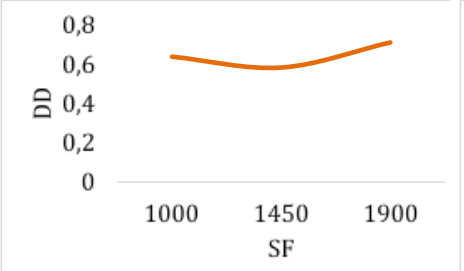

(c)

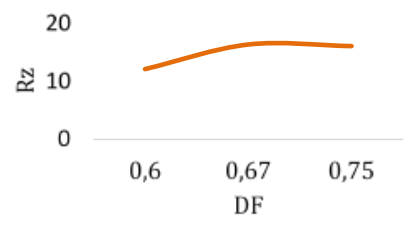

(e)

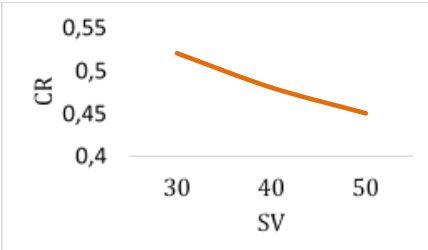

(g)

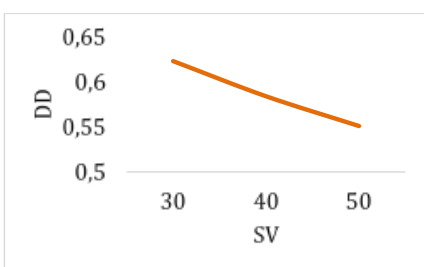

(i)

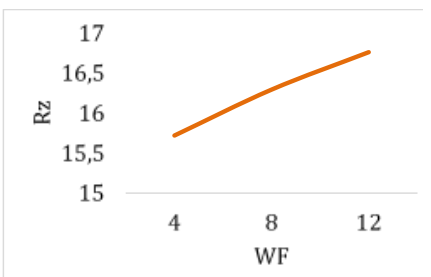

(k)

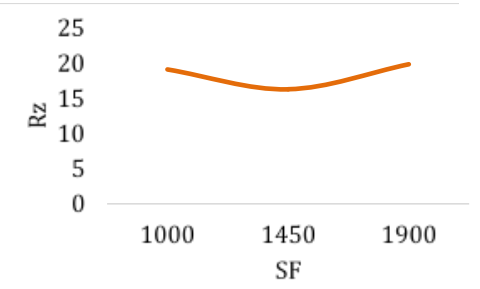

(b)

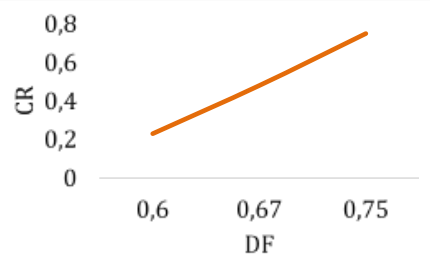

(d)

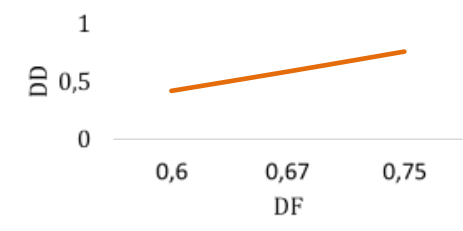

(f)

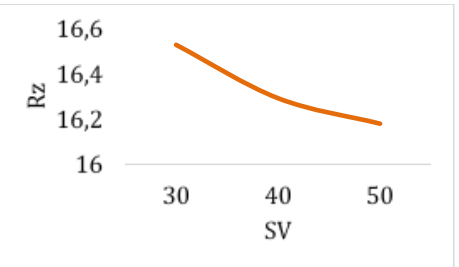

(h)

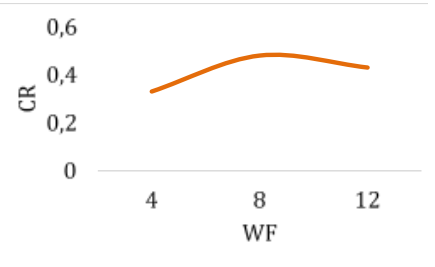

(j)

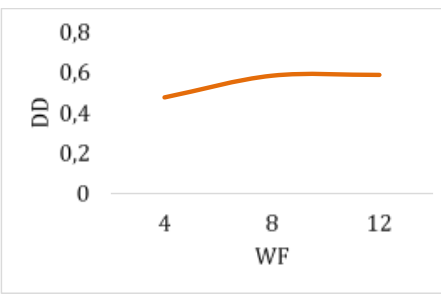

(1)

Fig. 2. Variation of Process Parameters (a) CR versus SF (b) Rz versus SF (c) DD versus SF (d) CR versus DF (e) Rz versus DF (f) DD versus DF (g) CR versus SV (h) Rz versus SV (i) DD versus SV (j) CR versus WF (k) Rz versus WF (l) DD versus WF. 
Fig. $2 \mathrm{~g}$-i show the variation of response characteristics with SV. It is evident form the Figures that smaller value SV is beneficial for CR, Rz and DD. Smaller value of SV (30V) is favourable for maximum $\mathrm{CR}$, minimum $\mathrm{Rz}$ and minimum DD. It is clear from Fig. $2 \mathrm{j}$ that medium value of WF $(8 \mathrm{~m} / \mathrm{min})$ gives maximum CR. However, with further increase in $\mathrm{WF}$ (up to $12 \mathrm{~m} / \mathrm{min}$ ), the CR value decreases. In case of Rz, smallest value of WF (4 m/min) suggests minimum $\mathrm{Rz}$ and with amplifying the WF, the Rz value ameliorates (Fig. 2k). It is also evident from Fig. 21 that the smaller value of WF $(4 \mathrm{~m} / \mathrm{min})$ is in the favour of minimum DD.

Empirical model developed for responses are given in Eq. (1), (2) and (3).

$$
\mathrm{CR}=-1.18302-2.94369 \mathrm{E}-003 * \mathrm{SF}+5.02963 * \mathrm{DF}+0.022153 * \mathrm{SV}+0.11784 * \mathrm{WF}-
$$

$0.038333 * \mathrm{DF} * \mathrm{SV}+1.03465 \mathrm{E}-006 * \mathrm{SF} 2-6.59274 \mathrm{E}-003 * \mathrm{WF} 2$

$\mathrm{Rz}=-148.31527-0.045791 * \mathrm{SF}+554.64432 * \mathrm{DF}+0.12999 * \mathrm{WF}+1.60727 \mathrm{E}-005 * \mathrm{SF} 2-$

$391.24875 * \mathrm{DF} 2$

$\mathrm{DD}=-1.44415-1.18151 \mathrm{E}-003 * \mathrm{SF}+3.91444 * \mathrm{DF}+0.024480 * \mathrm{SV}+0.068862 * \mathrm{WF}-$

$0.041583 * \mathrm{DF} * \mathrm{SV}+4.35046 \mathrm{E}-007 * \mathrm{SF} 2-3.43145 \mathrm{E}-003 * \mathrm{WF} 2$

A multi-objective grey wolf optimization (MOGWO) intelligent optimization technique has been used for WEDM parameters $[10,11]$. Table 4 gives the random solutions predicted by MOGWO and the experimental results of responses obtained after WEDM of gears at those optimum parameters. The suggested solutions are investigated empirically, due to the machine tool constraints. The suggested settings of input process parameters are in decimal, which may not be possible to set on machine tool. Thus, the process parameters initially set at some feasible values (which can be available on machine tool) and predicted solutions are calculated using Eq. 1, 2 and 3. It is observed that the values of responses obtained after experimentation have been in close agreement with the predicted solutions. Thus, the proposed approach can be efficiently applied for the multiple performance characteristics optimization of WEDM during the development of SS316 miniature gears. Fig. 3 shows the optical micrographs of miniature gears developed at optimized setting suggested by improved MOGWO.

Table 4. Results of optimization prediction, and confirmation tests

\begin{tabular}{|c|c|c|c|c|c|c|}
\hline \multirow{2}{*}{ Optimal conditions } & \multicolumn{3}{|c|}{ Predicted Responses characteristics } & \multicolumn{3}{|c|}{ Experimental Responses } \\
\cline { 2 - 7 } & $\mathrm{CR}$ & $\mathrm{Rz}$ & $\mathrm{DD}$ & $\mathrm{CR}$ & $\mathrm{Rz}$ & $\mathrm{DD}$ \\
\hline $\mathrm{A}_{1900} \mathrm{~B}_{0.75} \mathrm{C}_{33} \mathrm{D}_{10}$ & 1.033 & 19.909 & 0.9414 & 1.10 & 20.217 & 0.9503 \\
\hline $\mathrm{A}_{1350} \mathrm{~B}_{0.6} \mathrm{C}_{35} \mathrm{D}_{4}$ & 0.082 & 11.616 & 0.3064 & 0.087 & 11.882 & 0.3129 \\
\hline $\mathrm{A}_{1180} \mathrm{~B}_{0.6} \mathrm{C}_{37} \mathrm{D}_{4}$ & 0.136 & 12.487 & 0.3192 & 0.145 & 12.794 & 0.3241 \\
\hline
\end{tabular}

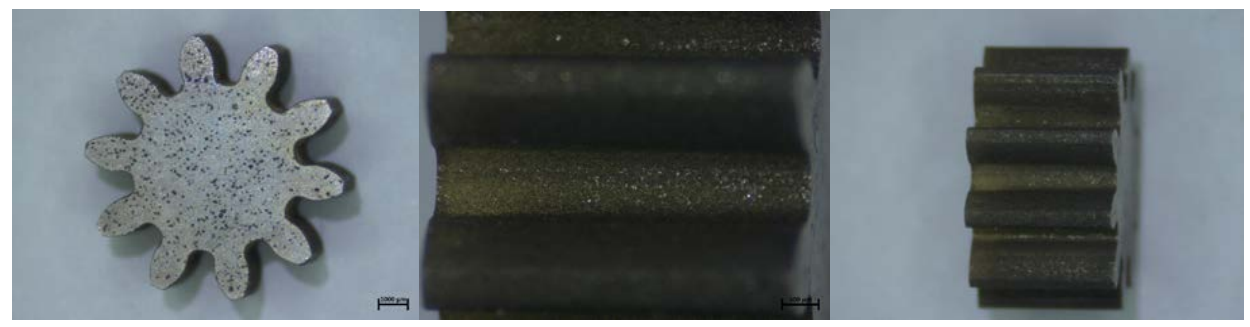

Fig. 3. Optical micrographs of the small gears fabricated by WEDM at optimum conditions.

\section{Conclusion}

The following conclusions are drawn from this work: 
- Successful fabrication of SS316 alloy miniature gear using WEDM. However, the quality obtained in terms of surface roughness and dimensional accuracy can further be improved with future attempts.

- A medium to high value of servo feed, low value of voltage and duty factor, and low to medium value of wire feed rate are required to secure the optimum responses. However, an optimum parameter setting is required.

- The best values of the responses obtained in the present work are, cutting rate- 1.033 $\mathrm{mm} / \mathrm{min}$, mean roughness depth- $11.882 \mu \mathrm{m}$, dimensional deviation- 0.3129 .

- $\quad$ ANOVA suggests that duty factor is the most contributing parameter which affects $\mathrm{CR}, \mathrm{Rz}$ and DD in the fabrication of SS316 alloy gear by WEDM.

\section{References}

1. N.K. Jain, S.K. Chaubey, Review of miniature gear manufacturing. In: Hashmi MSJ (ed.) Comprehensive materials finishing- 1, 504-538. Oxford: Elsevier (2016)

2. D.P. Townsend, Dudley's Gear Handbook; Tata McGraw-Hill, Publishing Company: New Delhi, India (2011)

3. J.R. Davis, Gear Materials, Properties, and Manufacture; ASM International, Ohio, USA (2005)

4. S.K. Chaubey, N.K. Jain, International Journal of Advanced Manufacturing Technology, 93, 9 (2017)

5. C. Anghel, K. Gupta, T.C. Jen, Optik - International Journal for Light and Electron Optics (Elsevier), 203, 164049 (2020)

6. T.C. Phokane, K. Gupta, C. Popa, On Abrasive Water Jet Machining of Miniature Brass Gears" (2018). In Proceedings of International Gear Conference, Lyon (France), Vol II, pp 384-392, Chartridge Books Oxford, ISBN 978-1-911033-43-1 (2018)

7. S. Di, R. Haung, G. Chi, Study on micro-machining by micro-WEDM. Proceedings of the 1st IEEE conference on nano/micro engineered and molecular systems, Zhuhai, China, 615-619 (2006)

8. M.Y. Ali, A.N.M. Karim, E.Y.T. Adesta, A.F. Ismail, A.A. Abdullah, M. Idris, Int J Precis Eng Manuf 11, 5 (2010)

9. D.G. Montgomery, Design and Analysis of Experiments. 7th ed. New Delhi: John Willey \& Sons, (2009)

10. S. Mirjalili, S.M. Mirjalili, A. Lewis, Adv. Eng. Soft. 69 (2014)

11. X.S. Yang, Nature-Inspired Metaheuristic Algorithms. 2nd ed. Luniver Press, Frome, UK (2010) 